\title{
Study on Leakage of Sesame (Sesamum indicum L.) and Coconut (Cocos nucifera L.) Liposomes
}

\author{
DWI HUDIYANTI ${ }^{1 *}$, TRI JOKO RAHARJO², NARSITO NARSITO² and SRI NOEGROHATI ${ }^{3}$ \\ 'Department of Chemistry, Faculty of Science and Mathematics, \\ Diponegoro University, Semarang, Indonesia. \\ 2Department of Chemistry, Faculty of Mathematics and Natural Sciences, \\ Gadjah Mada University, Yogyakarta, Indonesia \\ ${ }^{3}$ Faculty of Pharmacy, Gadjah Mada University, Yogyakarta, Indonesia \\ *Corresponding author E-mail: dwi_hudiyanti@undip.ac.id \\ http://dx.doi.org/10.13005/ojc/310152
}

(Received: November 30, 2014; Accepted: January 10, 2015)

\begin{abstract}
Leakage phenomena on sesame (Sesamum indicum L.) and coconut (Cocos nucifera L.) liposomes has been studied to evaluate their ability as drug delivery materials. Permeation of carboxyfluorescein through the liposomes with and without added cholesterol was examined. Sesame liposomes release carboxyfluorescein less than coconut liposomes in all circumstances. Sesame liposomes save about $50 \%$ of payload after 17 hours of storage while coconut liposomes only $10 \%$. Addition of cholesterol has increase storage capability of all liposomes. The sesamecholesterol and coconut-cholesterol liposomes save greater amount of payload compare to the original. Sesame liposomes have better potency as drug delivery systems.
\end{abstract}

Key words: coconut; cholesterol; drug delivery; leakage; liposome; sesame.

\section{INTRODUCTION}

Liposomes are important in medicinal, biotechnological, cosmetic, and industrial applications. They are used as delivery vehicles for drug or other active materials in their compartments. They have ability to encapsulate hydrophilic compounds within their interior cavity and carrying hydrophobic compounds in their membranes ${ }^{1,2}$. Since the discovery until 2008, there are some drugs have been approved for use in a liposomal delivery system 1 . There are also some cosmetics preparation that contain liposomes to encapsulate and carry the active ingredients to specific cells in the skin $^{3-5}$. The potential use of liposomes as carriers of active ingredients has attract a lot of attention and has been explored tremendously. This is because they are biodegradable, biocompatible, have low toxicity and do not activate the immune system $^{2}$. It is also because of their ability to bring 
active material directly to the active site, known as targeted delivery ${ }^{6}$. Research on liposomes from natural phospholipid that were derived from soybeans has been widely reported ${ }^{7-10}$ but research on liposomes of coconut and sesame phospholipids so far only a few ${ }^{11}$.

The most important property of liposomes that will influence their efficacy is permeability and leakage of liposomes. Leakage is a condition when liposomes release some of their payload to the environment spontaneously after they are produced. Leakage or permeability properties of phospholipid membranes of liposomes is key element in the ability of liposomes to store their payload in their cavity. It is also critical to drug transport across the cell membrane and the ability of drug to reach the target in intracellular sites ${ }^{12}$.

Membrane permeability and leakage are affected by cholesterol ${ }^{13,14}$. Cholesterol molecule is known as lipid with small head and large and rigid tail. It is an amphiphilic molecule and easily merges into the phospholipid bilayer. Cholesterol reduces water and ion permeability ${ }^{15-17}$, regulates $\mathrm{CO}_{2}$ permeability of cell membranes ${ }^{18}$ and other functions as well ${ }^{19,20}$.

The ability of liposomes to store their payload is indicated by the amount of leakage after certain time. 5-Carboxyfluorescein (CF) was used as payload ${ }^{21-23}$. The release of $\mathrm{CF}$ from liposomes was calculated by the following equation:

$$
L \%=\left(\frac{L_{4}-I_{0}}{I_{s}-I_{0}}\right) \times 100 \%
$$

L\% : Liposomes leakage.

1: intensity of $C F$ in liposomes at $t$ second.

$\mathrm{I}_{0}$ : intensity of $\mathrm{CF}$ in liposomes at 0 second.

I,: intensity of CF in liposomes after the liposomes were broken down.

This study investigate the potency of liposomes made from natural phospholipids, i.e., coconut meat (Cocos nucifera L.) and sesame seeds (Sesamum indicum L.) to be used as carrier for active substances in drug delivery system. These resources have been used as basic ingredients in Indonesian traditional medicine. These resources are quite abundant and empirically known as save materials and never cause any health problem for users. Soybean (Glycine max) phospholipids that have been used as basic ingredient of liposomes in various applications were selected as positive control.

\section{MATERIAL AND METHODS}

\section{Material and equipments}

Phospholipids of coconut and sesame were obtained in house from coconuts and sesame endosperm ${ }^{25}$. Soybean phospholipid $(30 \% \mathrm{~L}-\alpha-$ phosphatydilcholine, Cat no. P3644), Triton X-100 (cat no. X-100), cholesterol (cat no. 86826) and 5Carboxyfluorescein (cat no. C8667) were obtained from Sigma-Aldrich. Phosphate buffer solution (PBS) $10 \mathrm{mM} \mathrm{pH} 7.41$ was prepared from $\mathrm{Na}_{2} \mathrm{HPO}_{4} \cdot 2 \mathrm{H}_{2} \mathrm{O}$ (Merck) and $\mathrm{NaH}_{2} \mathrm{PO}_{4} \cdot 2 \mathrm{H}_{2} \mathrm{O}$ (Merck). Sephadex G 50 was from Pharmacia, Uppsala, Sweden.

Mini extruder was obtained from Avanti Polar Lipids. A polycarbonate membrane with pore size of $100 \mathrm{~nm}$ was placed inside the extruder. Leakage analysis was conducted on spectrofluorometer SPEX Fluorolog 2, Model F212, with computer system DMIB from SPEX Industries Inc., Edison, NJ.

\section{Liposomes preparation}

Liposomes of coconut, sesame and soybean were prepared by lipid hydration process as described by Hudiyanti et al. ${ }^{11}$. Sesame phospholipid (1 mmol) was diluted in $1 \mathrm{~mL}$ of chloroform / methanol $(1: 1 \mathrm{v} / \mathrm{v})$ in a test tube. The solvent was evaporated under flow of nitrogen gas to form thin film. Opened tube with thin layer was stored in a drying oven for 1 night. After 1 night the tube was taken from the drying oven, filled with nitrogen gas then covered and sealed with parafilm. The tube was deposited in a freezer until used.

To prepare liposomes $1 \mathrm{~mL}$ of PBS was added to the tube and agitated in 30 seconds. The tube then was immersed in liquid nitrogen until all the solution has been frozen. Subsequently the tubes was soaked in a water bath with temperature $50^{\circ} \mathrm{C}$ until all the solution melt. The tube was frozen back in liquid nitrogen. The procedure was repeated 
several times until thin film was completely dispersed. The phospholipid dispersion obtained was passed through an extruder. A Clear dispersion of liposomes was obtained. Dispersion of liposomes were then put into a refrigerator and allowed to stand for 1 night.

\section{Leakage analysis}

To determine the ability of liposome as carriers, an analysis of leakage was performed. Liposomes with CF payload were prepared. CF was added to the PBS solution so that the concentration was $100 \mathrm{mM}$. The solution was used as liposomes payload. CF-Liposome dispersions were prepared as above. CF-Liposome dispersions were then analysed in a spectrofluorometer. Excitation wavelength was set at $490 \mathrm{~nm}$, while the emission was at $520 \mathrm{~nm}$. The intensities were measured for 17 hours at temperature $25^{\circ} \mathrm{C}$ every $5 \mathrm{~s}$. At the end of the analysis, the liposomes were broken down using $100 \mathrm{mM}$ Triton X-100 solution to released all the payload into the PBS medium. The maximum intensity was measured. The effect of cholesterol was studied by adding cholesterol to liposomes preparation with varied concentration.

\section{RESULTS AND DISCUSSION}

In this study leakage analysis were conducted to determine the nature of liposomes that are closely linked to their function as carrier of active ingredients. Analysis was performed using $\mathrm{CF}$ as a model of the active ingredients. CF is a fluorescent probe that is soluble in polar medium. Leakage of liposomes indicated by the release of CF during observation. Leakage analysis in this study was carried out continuously for 17 hours.

The amount of leakage ( $L \%)$ of coconut, sesame and soybean liposomes are presented in Figure 1. Leakage pattern of coconut and sesame liposomes were similar to the soybean patterns. In general the liposomes leaked fast at the begining and became slower after 2 hours of observation. In 2 hours, coconut liposomes leaked $40 \%$ of the payload while sesame only $10 \%$. The analysis showed that the ability to store CF of coconut liposomes lower than sesame and soy liposomes. After 17 hours coconut liposome has released $88 \%$ of its payload, and sesame liposome was $42 \%$. By comparison soybean liposome was only $38 \%$. This results show that coconut liposomes have more defects or pores in their membrane phospholipid hence they leak more payload than sesame liposomes.

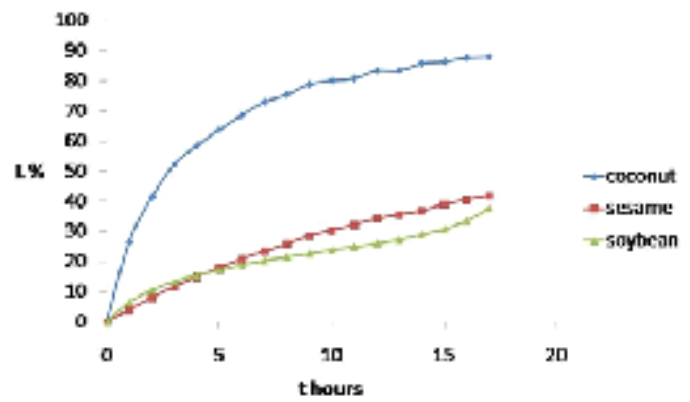

Fig. 1: Leakage of coconut, sesame and soybean liposomes during 17 hours observation

Further analysis was conducted to determine the effects of adding cholesterol to the leakage. It is known that the addition of cholesterol has effect on membrane permeability ${ }^{13,15,18,19,24}$. In turn the liposomes leakage is closely related to the permeability properties of its phospholipid bilayer membrane.

The addition of cholesterol to the coconut, sesame and soybean liposomes showed decline of the leakage of all liposomes as shown in Figure 2, 3, and 4. The cholesterol concentrations also influenced the amount of $\mathrm{CF}$ released. The greater the cholesterol concentration the more reduced the leakage. There is a decrease in the rate of leakage with increasing concentrations of cholesterol (Figure 5). In general, the highest liposome leakage rate occurred at cholesterol concentration less than $40 \%$.

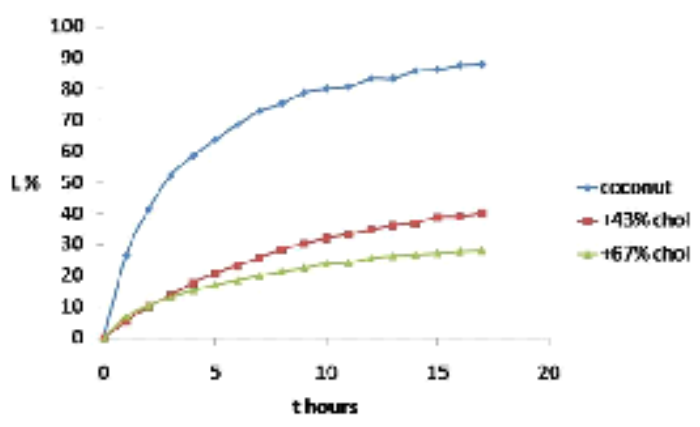

Fig. 2: Leakage of coconut liposomes with added cholesterol during 17 hours observation 


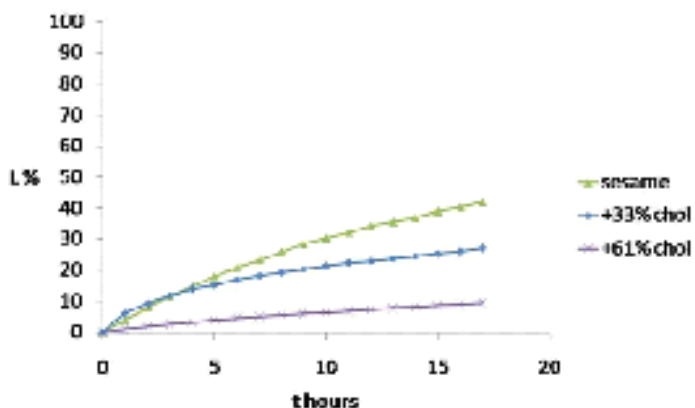

Fig. 3: Leakage of sesame liposomes with added cholesterol during 17 hours observation

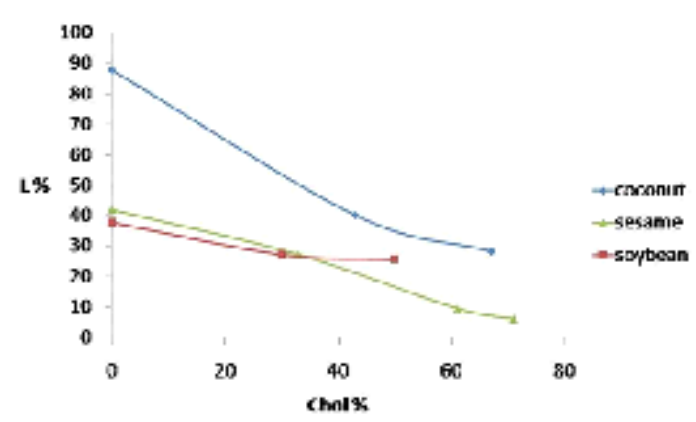

Fig. 5: Leakage of coconut, sesame and soybean liposomes after 17 hours observation as a function of cholesterol concentration

These results suggest that sesame liposomes have better potency to be used as carrier of hydrophilic substance than coconut liposomes. Adding cholesterol to the preparation has reduced the leakage of all liposomes. Cholesterol has greater effect on coconut liposomes. Addition of cholesterol to coconut liposomes reduces the leakage more effectively than to sesame liposomes. The molecular species in coconut phospholipids mostly are phosphatydilserine and phosphatydilethanolamine ${ }^{25}$. The presence of

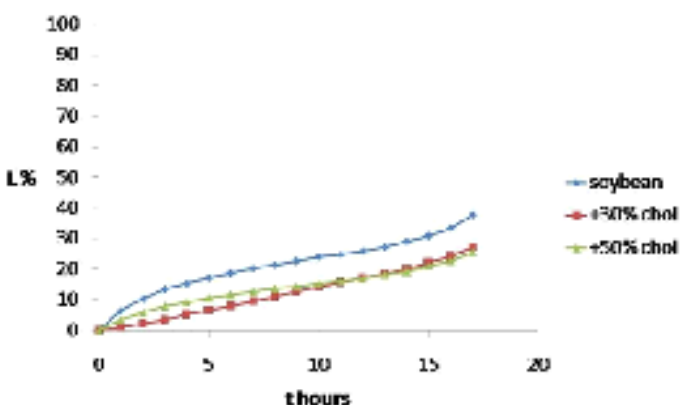

Fig. 4: Leakage of soybean liposomes with added cholesterol during 17 hours observation

cholesterol might have promoted hydrogen bonding formation among the phospholipids molecular species on the bilayer surface, which could hinder the membrane pore formation.

\section{CONCLUSION}

Sesame liposomes save about $50 \%$ of payload after 17 hours of storage while coconut liposomes only $10 \%$. Addition of cholesterol has increase storage capability of both sesame and coconut liposomes. The results above illustrate the possibility of using sesame and coconut liposomes. Sesame liposomes have better potential application as drug delivery materials.

\section{ACKNOWLEDGEMENTS}

Dwi Hudiyanti would like to express her gratitude to Prof. K. Edwards for fruitful discussion and an opportunity to work in her laboratory, Dr. V. A. Hernandez for his help with the SPEX. This study was supported in part by Sandwich-Like Program Dikti Indonesia.

\section{REFERENCES}

1. Slingerland, M; Guchelaar, H-J; Gelderblom, 4. H. Drug Discov. Today 2012, 17,160-166

2. Torchilin, V.P. Nat. Rev. Drug Discov. 2005, 4, 145-160

3. Laouini, A.; Jaafar-Maalej, C.; LimayemBlouza, I.; Sfar, S.; Charcosset, C.; Fessi, H. J. Colloid Sci. Biotechnol. 2012, 1, 147-168
Mehl, T. L. S.; Zaias, N. 2001, US patent 6, 183, 451

5. Guaratini, T.; Gianeti, M. D.; Campos P. M. B. G. M. Int. J. Pharm. 2006, 327, 12-16

6. Allen, T. M.; Cullis, P. R.; Adv. Drug Deliv. Rev. 2013, 65, 36-48

7. Aisha, A. F.; Majid, A. M. S. A.; Ismail, Z. BMC 
Biotechnol. 2014, 14, 23

8. Chen, Y.; Wu, Q.; Zhang, Z.; Yuan, L.; Liu, X.; Zhou, L. Molecules 2012, 17, 5972-5987

9. Yokota, D.; Moraes, M.; Pinho, S. C. Braz. J. Chem. Eng. 2012, 29, 325-335

10. Imura, T.; Otake, K.; Hashimoto, S.; Gotoh, T.; Yuasa, M.; Yokoyama, S.; Sakai, H.; Rathman, J. F.; Abe, M.; Colloids Surf. B Biointerfaces 2003, 27, 133-140

11. Hudiyanti, D.; Raharjo, T. J.; Narsito, N.; Noegrohati, S. Indo. J. Chem. 2012 12, 5761

12. Mouritsen, O. G.; Jørgensen, K. Pharm. Res. 1998, 15, 1507-1519

13. Papahadjopoulos, D.; Nir, S.; Ohki, S. Biochim. Biophys. Acta BBA - Biomembr. 1972, 266, 561-583

14. De Kruyff, B.; Demel, R. A.; Deenen, L. L. M. Biochim. Biophys. Acta BBA - Biomembr. 1972, 255, 331-347

15. Matsuoka, S.; Murata, M. Biochim. Biophys. Acta. 2002, 1564, 429-434

16. Saito, H.; Shinoda, W.; J. Phys. Chem. B. 2011, 115, 15241-15250

17. Mathai, J. C.; Tristram-Nagle, S.; Nagle, J. F.;
Zeidel, M. L. J. Gen. Physiol. 2008, 131, 6976

18. Itel, F.; Al-Samir, S.; Öberg, F.; Chami, M.; Kumar, M.; Supuran, C. T.; Deen, P. M. T.; Meier, W.; Hedfalk, K.; Gros, G.; Endeward, V. FASEB J. 2012, 26, 5182-5191

19. Papahadjopoulos, D.; Cowden, M.; Kimelberg, H. Biochim. Biophys. Acta BBA Biomembr. 1973, 330, 8-26

20. López-Pinto, J.M.; González-Rodríguez, M.L.; Rabasco, A.M. Int. J. Pharm. 2005 298, $1-12$

21. Chen, R.F.; Knutson, J.R. Anal. Biochem. 1988, 172, 61-77

22. Hashizaki, K.; Taguchi, H,; Sakai, H.; Abe, M.; Saito, Y.; Ogawa, N. Chem. Pharm. Bull. 2006, 54, 80-84

23. Barbet, J.; Machy, P.; Truneh, A.; Leserman, L. D. Biochim. Biophys. Acta BBA Biomembr. 1984, 772, 347-356

24. Finkelstein, A.; Cass, A. Nature 1967, 216, 717-718

25. Hudiyanti, D.; Raharjo, T. J.; Narsito, N.; Noegrohati, S. J. Agritech. Fak. Teknol. Pertan. UGM. 2012, 32, 01 